\title{
Lesson of the month 2: Blunt abdominal trauma: atypical presentation of phaeochromocytoma
}

\author{
Authors: Sarah Faloon, ${ }^{\mathrm{A}}$ Hema Venkataraman, ${ }^{\mathrm{B}}$ Kassiani Skordilis, ${ }^{\mathrm{C}}$ Ewen A Griffiths, ${ }^{\mathrm{D}}$ Neil JL Gittoes, ${ }^{\mathrm{E}}$ \\ Zaki K Hassan-Smith ${ }^{\mathrm{F}, \mathrm{G}}$ and John Ayuk $^{\mathrm{H}}$
}

\begin{abstract}
A 26-year-old man presented following blunt abdominal trauma to a regional major trauma centre for emergency embolisation of a retroperitoneal bleed from a presumed renal laceration. Imaging had also revealed a large right suprarenal mass. Embolisation resulted in a hypertensive crisis raising the suspicion of a metabolically active adrenal tumour. The course was further complicated by the development of ischaemic bowel requiring emergency laparotomy. Intraoperatively he became haemodynamically unstable from an actively haemorrhaging lesion. Emergency laparotomy and adrenalectomy was performed as a life-saving procedure. Histology confirmed a phaeochromocytoma. The patient made a gradual recovery and was discharged home with no sequelae. Definitive management of phaeochromocytoma is surgical resection which requires prolonged preoperative optimisation with alpha receptor blockers to adequately control blood pressure and prevent hypertensive crises. Parenteral alpha receptor blockers, such as phentolamine, are optimal treatment for intraoperative hypertensive emergencies, yet they are currently not available in the UK.
\end{abstract}

KEYWORDS: Phaeochromocytoma, abdominal trauma, interventional radiology

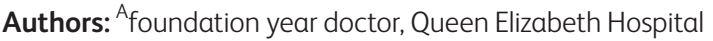
Birmingham, University Hospitals Birmingham NHS Foundation Trust, Birmingham, UK; ${ }^{\mathrm{B}}$ specialist registrar, Queen Elizabeth Hospital Birmingham, University Hospitals Birmingham NHS Foundation Trust, Birmingham, UK; ${ }^{C}$ consultant pathologist, Queen Elizabeth Hospital Birmingham, University Hospitals Birmingham NHS Foundation Trust, Birmingham, UK; ${ }^{\mathrm{D}}$ consultant upper GI surgeon, Queen Elizabeth Hospital Birmingham, University Hospitals Birmingham NHS Foundation Trust, Birmingham, UK; E consultant and professor of endocrinology, Queen Elizabeth Hospital Birmingham, University Hospitals Birmingham NHS Foundation Trust, Birmingham, UK; F consultant endocrinologist, Queen Elizabeth Hospital Birmingham, University Hospitals Birmingham NHS Foundation Trust, Birmingham, UK; ${ }^{G}$ visiting professor, Coventry University, UK; ${ }^{\mathrm{H}}$ consultant endocrinologist, Queen Elizabeth Hospital Birmingham, University Hospitals Birmingham NHS Foundation Trust, Birmingham, UK

\section{Case presentation}

A 26-year-old man presented as a major trauma patient to a regional centre for emergency embolisation of an ongoing retroperitoneal bleed from a presumed renal laceration. He had sustained blunt abdominal trauma after falling from a height onto a metal railing. He had been previously investigated by his GP for episodes of severe headache and palpitations precipitated by alcohol and exercise. He had been diagnosed with migraine following normal investigations including computed tomography (CT) head and Holter monitor.

$\mathrm{CT}$ imaging demonstrated a large right suprarenal tumour and retroperitoneal haematoma, alongside a renal laceration. He proceeded to have a selective aortic and renal angiogram and guided embolisation. During this procedure two tumoural vessels arising from the anterior aorta revealed extensive abnormal vascularity, but no obvious extravasation. At this point the patient became quite hypertensive and tachycardic raising the possibility of a metabolically active tumour. Therefore, as the bleeding seemed to have stopped, tumoural blood supply was not interrogated further.

This was followed by a period of sustained hypotension, requiring ongoing management on the intensive care unit. In view of the clinical course and in the presence of a suprarenal lesion, a phaeochromocytoma was suspected. He remained hemodynamically unstable requiring intubation, inotropic support and haemodiafiltration. He demonstrated an extremely labile blood pressure with worsening liver and renal function, rising lactate and dropping haemoglobin.

CT on day 3 (Fig 1) showed ongoing blood loss and a significant area of gangrenous infarction within the ascending and transverse colon. Following extensive multidisciplinary discussions, the patient underwent emergency laparotomy for bowel resection, with a plan to perform adrenalectomy at a later date, following preoperative alpha receptor blockade. Persistent hypotension, non-availability of parenteral alpha receptor blockers and need for emergency surgery precluded adequate preoperative optimisation of the catecholamine excess state. The patient was unable to receive medications via an enteral route due to the infarcted bowel and nil by mouth status. Plasma metanephrine results were awaited from another centre and hence unavailable at the time of surgery.

At emergency laparotomy, an ischaemic ascending colon (Fig 2) with patchy gangrenous areas was found in relating of the 




Fig 1. CT abdomen showing large residual haematoma. $C T=$ computed tomography

expanding retroperitoneal haematoma which was compromising the mesenteric circulation. An emergency right hemicolectomy was performed with the ends left stapled off. As there was significant retroperitoneal bleeding of over $1,000 \mathrm{~mL}$ from the adrenal mass, an emergency right adrenalectomy was also performed as a life-saving step to arrest further blood loss. The retroperitoneum was packed and the abdomen was temporarily closed. A relook was performed 48 hours later and the packs removed and a small bowel to transverse colon anastomosis was performed to restore intestinal continuity.

Macroscopically, the right adrenal gland consisted of a firm mass, $500 \mathrm{~g}$ in weight, measuring $15 \mathrm{~cm} \times 15 \mathrm{~cm} \times 10 \mathrm{~cm}$ with evidence of rupture (Fig 3). Histological examination confirmed a phaeochromocytoma with tumour cells staining positive for synaptophysin and chromogranin (Fig $4 \mathrm{a}$ ) and a Phaeochromocytoma of the Adrenal gland Scaled Score (PASS) of 8 (Fig 4b), suggestive of a biologically aggressive tumour, but Ki-67 $<1 \%$ consistent with an indolent tumour. SDHB expression was preserved (Fig 4C)

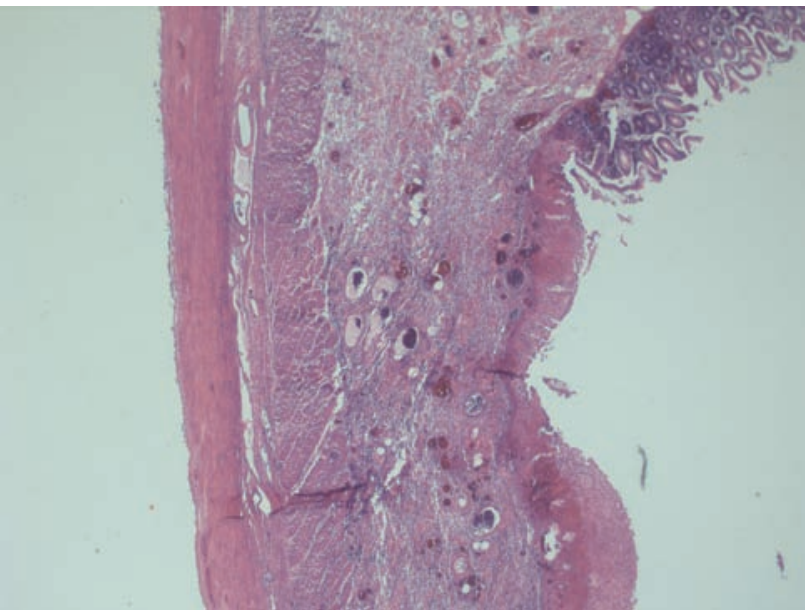

Fig 2. Colonic section showing necrosis of mucosa.



Fig 3. Ruptured adrenal tumour.

Subsequent plasma metanephrine results were consistent with a diagnosis of phaeochromocytoma. Postoperatively his plasma normetanephrine level fell from $>25,000 \mathrm{pmol} / \mathrm{L}$ to $270 \mathrm{pmol} / \mathrm{L}$.

He made a gradual recovery and was discharged home with no sequelae. Follow-up CT scan showed interval improvement with almost complete resolution of right pararenal haematoma. Metaiodobenzylguanidine (MIBG) iodine 123 scan found no evidence of recurrent or macroscopic metastatic disease. Clinical presentation, histology and radiology were discussed at the adrenal multidisciplinary team meeting and he was referred to the clinical genetics service for screening for familial phaeochromocytoma paraganglioma syndromes.

\section{Discussion}

This case is unique in the published literature in that it describes a successful outcome from emergency surgery for phaeochromocytoma without prior alpha receptor blockade, in a patient presenting following acute abdominal trauma.

This case demonstrates that interventional procedures are potential precipitants of crisis in patients with occult phaeochromocytoma. Dinkelaar et al discuss the case of a 63-yearold man who developed fatal complications from undiagnosed phaeochromocytoma provoked by splenic artery embolisation. ${ }^{1}$ Conversely, successful embolisation procedures have been described in patients with known phaeochromocytoma for neoadjuvant indications. ${ }^{2,3}$ In both cases, the patient was preoperatively managed with alpha blockers, and phentolamine was available.

The definitive management of phaeochromocytoma is surgical resection, which requires prolonged preoperative preparation with alpha receptor blockade to prevent intraoperative hypertensive crises. This is achieved with initial alpha-blockade, with subsequent beta-blockade for control of heart rate if required, usually for a minimum of 14 days. ${ }^{4}$ This is important to avoid precipitating a catecholamine-induced crisis during intraoperative handling of the tumour, eg hypertensive crisis and arrhythmias. ${ }^{5}$ Emergency adrenalectomy for phaeochromocytoma is rarely necessary and carries high morbidity and mortality, with mortality reported as high as $45 \%$ prior to the introduction of routine preoperative alpha-blockade. $^{5-7}$ 

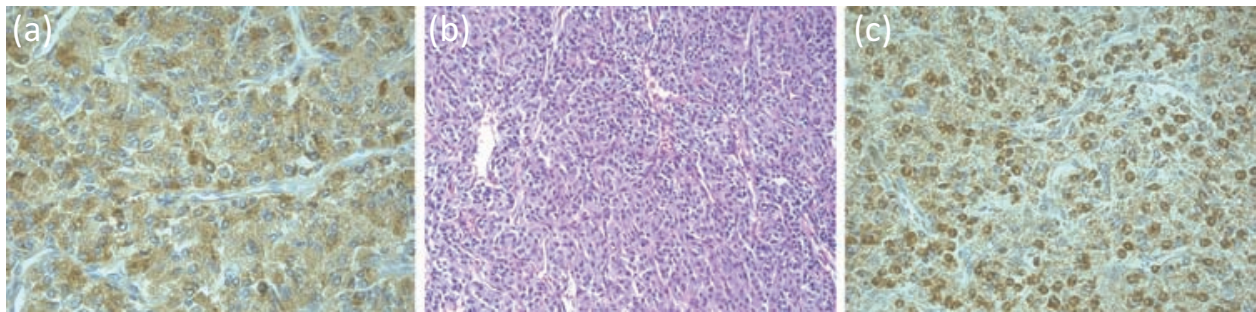

Fig 4. Images of the phaeochromocytoma. (a) Chromogranin staining. (b) Haematoxylin and Eosin section. (c) SDHB expression.

If a hypertensive crisis was to arise during surgery, this can be managed with infusion or boluses of phentolamine. ${ }^{8}$ Phentolamine is a short-acting reversible non-selective alphaadrenergic antagonist and is the optimal treatment for hypertensive emergencies. ${ }^{8}$ However, it is currently not available in the UK and was not available for the surgery performed in our case. Alliance Pharmaceuticals / Novartis discontinued its UK production in August 2012 and, according to the Specialist Pharmacy Service, there are currently no alternative injectable alpha blockers available within the UK; importers are experiencing difficulties sourcing formulations of either phentolamine or the alternative agent phenoxybenzamine. ${ }^{9}$

\section{Learning points}

> Suprarenal and adrenal tumours should not be embolised unless a diagnosis of phaeochromocytoma has been excluded, as this can precipitate a life-threatening crisis

> In cases of emergency phaeochromocytoma surgery, parenteral alpha receptor blockers are currently not readily available within the UK; patients should be managed with other intravenous antihypertensive agents

> In the absence of pre-operative alpha receptor blockade, teams must be prepared for sudden intra-operative cardiovascular complications

> Early biochemical analysis with plasma metanephrine testing is recommended in the diagnosis of phaeochromocytoma, but this is limited by slow turnaround times and lack of widespread availability for this test.

\section{Consent}

Consent to publish this article has been obtained from the patient.

\section{References}

1 Dinkelaar W, Elgersma O, Levin MD. A fatal complication caused by occult pheochromocytoma after splenic artery embolization for malignant hypersplenism. Cardiovasc Intervent Radiol 2012;35:971-4.

2 Jacob M, Macwana S, Vivekanand D. Anaesthetic management of a case of adrenal and extra-adrenal phaeochromocytoma for preoperative embolisation. Indian J Anaesth 2015;59:196.

3 Vagner H, Hey TM, Elle B, Jensen MK. Embolisation of pheochromocytoma to stabilise and wean a patient in cardiogenic shock from emergency extracorporeal life support. BMJ Case Rep 2015:pii:bcr2014206069.

4 Lenders JW, Duh QY, Eisenhofer G et al. Pheochromocytoma and paraganglioma: an endocrine society clinical practice guideline. J Clin Endocrinol Metab 2014;99:1915-42.

5 Scholten A, Cisco RM, Vriens MR et al. Pheochromocytoma crisis is not a surgical emergency. J Clin Endocrinol Metab 2013;98:581-91.

6 Hanna JS, Spencer PJ, Savopoulou C, Kwasnik E, Askari R. Spontaneous adrenal pheochromocytoma rupture complicated by intraperitoneal hemorrhage and shock. World J Emerg Surg 2011;6:27.

7 Bajwa SS, Bajwa SK. Implications and considerations during pheochromocytoma resection: A challenge to the anesthesiologist. Indian J Endocrinol Metab 2011;15:337-44.

8 Connor D, Boumphrey S. Perioperative care of phaeochromocytoma. BJA Education 2016;16:153-8.

9 Service Specialist Pharmacy. Shortage Memo Phentolamine and Phenoxybenzamine. London and South East Regional Medicines Information Service, 2014. www.sps.nhs.uk/articles/shortage-ofphentolamine-mesilate-injection/ [Accessed 25 March 2018].

Address for correspondence: Dr Sarah Faloon, Department of Endocrinology, 3rd Floor Heritage Building, Division of Medicine, Queen Elizabeth Hospital Birmingham, University Hospitals Birmingham NHS Foundation Trust, Birmingham B15 2TH, UK. Email: sarah.faloon@nhs.net 